\title{
Warm Winds in the Seyfert 1 Galaxy NGC 5548
}

\author{
K. C. Steenbrugge and J. S. Kaastra \\ SRON National Institute for Space Research, Sorbonnelaan 2, 3584 CA \\ Utrecht, The Netherlands
}

\begin{abstract}
We present the results of a recent long XMM-Newton and Chandra observation on the Seyfert 1 galaxy NGC 5548. This galaxy has a warm absorber with a wide range of ionization stages. The detection of $\mathrm{O}$ VI in both the UV and X-rays allows for a direct comparison of the measured column density in both wavelength bands. We also comment on the detection of $\mathrm{O} \mathrm{V}$, and its newly measured wavelength.
\end{abstract}

\section{Introduction}

The immediate environment of active galactic nuclei (AGN) is poorly understood. Possibly powered by radiation pressure, an outflowing wind is formed from the accretion disk. In some AGN, the wind is visible through its continuum and line absorption of the radiation from the nucleus. Studying these outflows is important in the study of the enrichment of the Inter Galactic Medium (IGM) as well as the accretion physics of gas onto a supermassive black hole.

NGC 5548, a Seyfert 1 galaxy, has been well studied in the X-rays due to its relative $\mathrm{X}$-ray brightness, and the fact that it is a nearby active galactic nucleus with a redshift of $\mathrm{z}=0.01676$ (Crenshaw \& Kraemer 1999). Done et al. (1995) detected an O VII and O VIII K-shell absorption edge using ROSAT data. Therefore, NGC 5548 is an ideal Seyfert 1 galaxy to study with the high resolution spectroscopy instruments onboard XMM-Newton and Chandra.

\section{Complex O VII triplet spectral region}

Fig. $1 \& 2$ show the spectral region around the O VII triplet. Note the strong O VII forbidden emission line, while the O VII intercombination line is not detected. Probably the O VII intercombination line is blended with an O VI line, which has a rest wavelength of $21.79 \AA$. The measured column density from the O VI absorption line at $22.01 \AA$ allows for a direct comparison with the column density measured in the UV band. The measured column density is an order of magnitude larger in the X-ray band than in the UV band (Arav et al. 2003). Other low ionization ions also have larger derived total column densities in the X-rays than in the UV band (Steenbrugge et al. 2003, 2004).

There is a strong $\mathrm{O} \mathrm{V}$ absorption line, indicating that the X-ray warm absorber spans at least part of the ionization stages observed in the UV. In our fit to the RGS spectrum (Steenbrugge et al. 2003) we used for this line $\lambda=$ 
$22.33 \AA$ as calculated using the HULLAC code (Behar, priv. comm.). For the later LETGS observation we used $\lambda=22.374 \AA$, as measured by Schmidt \& Beiersdorfer (priv. comm.) using the University of California EBIT-I electron beam ion trap (Beiersdorfer 2003). The difference between both wavelengths is similar to the FWHM of the LETGS. This difference in wavelength gives a good indication of the present uncertainties in the atomic data for the inner-shell lines.

From the LETGS data one can determine the broadening of the deeper lines, the O VII and O V lines in Fig. 2, and conclude that the broadening and measured outflow velocity are consistent with the five outflow velocities measured in the UV. This is a further indication that the UV and X-ray observations detect the same outflowing absorber. Our data also show that the five absorbers all have a large range in ionization stages, from lowly ionized Si IV through highly ionized O VIII.
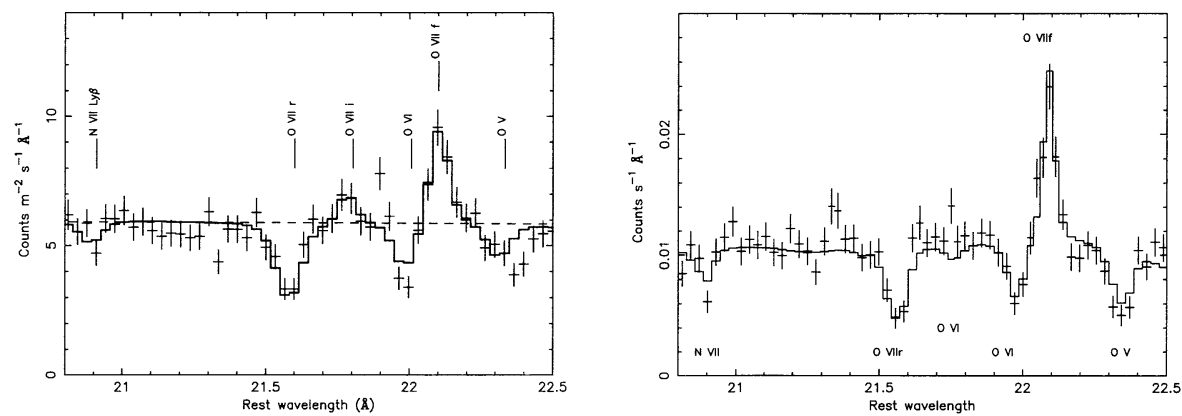

Figure 1. Left: RGS spectrum showing the O VII triplet. Right Same, for the LETGS spectrum.

Acknowledgments. This work is based on data obtained with $X M M-$ Newton, an ESA science mission with instruments and contributions directly funded by ESA Member States and the USA (NASA). SRON National Institute for Space Research is supported financially by NWO, the Netherlands Organization for Scientific Research.

\section{References}

Arav, N., et al. 2003, ApJ, 590, 174

Behar, E., private communication

Beiersdorfer, P. 2003, ARA\&A, 41, 343

Crenshaw, D. M., \& Kraemer, S. B. 1999, ApJ, 521, 57

Done, C., Pounds, K. A., Nandra, K., \& Fabian, A. C. 1995, MNRAS, 275, 417

Schmidt, M. \& Beiersdorfer, P., private communication

Steenbrugge, K. C., Kaastra, J. S., de Vries, C. P. \& Edelson, R. 2003, A\&A, 402,477

Steenbrugge, K. C., et al. 2004, in prep. 